\title{
From the bankruptcy problem and its Concede-and-Divide solution to the assignment problem and its Fair Division solution
}

\author{
Christian Trudeau (University of Windsor)
}

Working paper $15-06$

Working papers are in draft form. This working paper is distributed for purposes of comment and discussion only. It may not be reproduced without permission of the copyright holder. Copies of working papers are available from the author or at http://ideas.repec.org/s/wis/wpaper.html. 


\title{
From the bankruptcy problem and its Concede-and-Divide solution to the assignment problem and its Fair Division solution*
}

\author{
Christian Trudeau ${ }^{\dagger}$
}

April 21, 2016

\begin{abstract}
We revisit two classic problems: the assignment problem, in which agents create value when matched with a partner, and the bankruptcy problem, in which we need to share an endowment among agents with conflicting claims. We show that since Core Selection constrains us to exactly divide the value created by a pair of matched agents, the assignment problem can be seen as a two-player bankruptcy problem. This interpretation allows us to show that the classic Concedeand-Divide (Aumann and Maschler, 1985) sharing method for the bankruptcy problem is equivalent to the Fair Division solution (Thompson, 1981) for the assignment problem, itself the average of the extreme points of the core of Demange (1982) and Leonard (1983).

We then exploit the link between the two problems to offer two characterizations of the Fair Division solution. The key property is an adapation of the Minimal Rights First property (Curiel, Maschler and Tijs, 1987) for the bankruptcy problem. The minimal rights of a claimant is what is left of the endowment, if any, when all claimants but himself have received their full claims. The property states that we obtain the same shares if we distribute the minimal rights first, adjust the claims and endowment and proceed on the reduced problem or simply ignore them and proceed on the original problem. In assignment problems, the conceptual equivalent of minimal rights are the minimal core allocations.

Given the important role that minimal core allocations play in this link between assignment and bankruptcy problems, it is important to be able to compute them efficiently. We provide a new algorithm to compute them.
\end{abstract}

$J E L$ classification numbers: C71, D63

Keywords: assignment problems, bankruptcy problems, core, fair division, concede-and-divide, minimal rights.

\section{Introduction}

We revisit two classic sharing problems, the assignment and bankruptcy problems. While the two are well-studied, we provide, to our knowledge, the first link between the two problems. The link allows us to show the concordance of two well-known sharing methods. The link also allows for axioms and characterizations for the bankruptcy problem to spill over to the assignment problem.

\footnotetext{
*Special thanks to Marina Nunez for precious comments. The author also benefited from comments from Andrew Mackenzie and seminar participants at University of Barcelona and University of Vigo. A previous version circulated under the title "Minimal core allocations, empty threats and the Fair Division solution for the assignment problem".

${ }^{\dagger}$ Department of Economics, University of Windsor, 401 Sunset Avenue, Windsor, Ontario, Canada. Email: trudeauc@uwindsor.ca
} 
Assignment problems are well-studied: they represent a two-sided matching market, with buyers on one side and sellers on the other. They trade a good in indivisible units. Side payments are allowed and utility is measure in terms of money. Each buyer demands one unit, while sellers each have one unit. Goods are not identical (houses for example) and buyers have different evaluations of the units up for sale. Sellers have different reservation prices. The joint profit of matching a buyer with a seller is thus the difference between the evaluation of the buyer and the reserve price of the seller.

A natural way to define a cooperative game from this problem is to suppose that a coalition of buyers and sellers aim to maximize the joint total profits created by these trades. We know since Shapley and Shubik (1971) that the game has a non-empty core. There has been extensive studies of the game (see Roth and Sotomayor (1992) for an overview of the assignment problems, as a special case of two-sided matching problems) and its core (Balinski and Gale (1987), Hamers et al. (2002), Nunez and Rafels (2003)).

A bankruptcy problem consists in sharing an endowment among agents who have claims for it, with the sum of the claims larger than the endowment. While introduced in the economic literature by O'Neill (1982), the topic has been studied by religious and philosophical scholars for centuries, with many solutions studied by economists having been proposed as far back as in the 14th century. See Thomson $(2003,2015)$ for reviews of the literature.

For the assignment problem very few allocation methods, which tells us how much money to allocate to each agent for any given problem, have been studied. Among them, van den Brink and Pinter (2015) examine and characterize the Shapley value, which might not be in the core. Nunez and Rafels (2006) provide an allocation method that is always in the core, by first transforming the value matrix to make sure that the Shapley value of the corresponding game is always in the core. Adjustments to individual shares are then made, using the information on how we modified the matrix.

Thompson (1981) introduced the Fair Division solution as the average of two extreme core allocations, that are the most favorable (jointly) for either all buyers or all sellers. Demange (1982) and Leonard (1983) studied these extreme core allocations from a non-cooperative point of view (more precisely their strategyproofness). The solution is further studied in Nunez and Rafels (2002). It is shown that it satisfies two natural monotonicity property (if the value of the match between two agents increases, all else equal, the two agents do not suffer, and if an agent is added to the market, agents on the same side cannot gain while agents on the other side of the market cannot suffer). The solution also coincides with the $\tau$-value, a concept defined for general cooperative games by Tijs (1981), which consists in compromising between an utopia allocation, where all agents get their most favorable allocation compatible with the core, and a minimal-rights vector, where an agent receives what is left after all other members of a coalition have been paid their utopia allocation.

For the bankruptcy problem, proposed methods include the Proportional method (endowment shared in proportion to claims), the Constrained Equal Awards method (endowment shared as equally as possible, with no agent receiving more than her claim), the Constrained Equal Losses method (endowment shared such that the losses - claims minus shares - are as equal as possible, with no agent receiving a negative share) and the Random Arrival method (equivalent of the Shapley value (O'Neill (1982)). Methods combining in some ways the aforementioned methods have also been proposed, among them the Talmud method (Aumann and Maschler (1985)). For the case of two claimants, an interesting method is the Concede-and-Divide solution (Aumann and Maschler (1985)), which consists in first assigning to each agent his minimal right, which consists in what his left, if any, when the other agent receives his full claim. Whatever is left is then divided equally among agents.

We link the two problems in the following way. It is known since Shapley and Shubik that in the assignment problem, in any core allocation, if two agents are matched to each other, then their combined share is exactly equal to the value they create together. The problem is thus similar to a two-claimant bankruptcy problem, with the endowment equal to the value they create. As for the claims, we use the maximal core allocations. Our first result is that the prescriptions of the Concedeand-Divide solution coincide with those of the Fair Division solution.

Next, since the maximal and minimal core allocations for the assignment problems are key to the link with the bankruptcy problems, we provide a new method to obtain these values. We then obtain 
characterizations of the Fair Division solution that use both the link with the assignment problems, notably through some translation of axioms used in the bankruptcy context, and the method to extract minimal core allocations. Our key axiom is an adaptation of the Minimal Rights First property (Curiel, Maschler and Tijs (1987)). The axiom states that assigning the minimal rights first, adjusting the endowment and sharing what is left should give the same allocations as if we proceed directly from the original problem.

The paper is divided as follows. In Section 2, we introduce the assignment problem. The bankruptcy problem is defined in Section 3, which also offers a formal link with the assignment problem, and between the Fair Division solution for the assignment problem and the Concede-and-Divide solution for the bankruptcy problem. Theses links use minimal core allocations, and a new algorithm to calculate those is provided in Section 4. We offer characterizations of the Fair Division solution in Section 5. Various discussions are offered in Section 6, notably the similarities between the Fair Division solution and the one proposed by Nunez and Rafels (2006).

\section{The assignment problem}

We have two sets of agents, $N$ and $N^{\prime}$. Without loss of generality, we can assume that $|N|=\left|N^{\prime}\right|$, with $N=\{1,2, \ldots, n\}$ and $N^{\prime}=\left\{1^{\prime}, 2^{\prime}, \ldots, n^{\prime}\right\}$. For each $i \in N$ and $j^{\prime} \in N^{\prime}$, we have a value $v_{i j^{\prime}} \geq 0$ that we obtain if we match these two agents. Let $v=\left(v_{i j^{\prime}}\right)_{i \in N, j^{\prime} \in N^{\prime}}$ be the vector of all pairwise values. We slightly abuse language and call $v$ a (value) matrix.

An assignment problem is $A=\left(N, N^{\prime}, v\right)$, the sets of players and the set of matching values. When there is no risk of confusion, we simply identify a problem by $v$, its value matrix.

We then have an assignment problem to solve: An eligible assignment is a set of pairs $a$ such that if $\left(i, j^{\prime}\right) \in a$, there is no $k^{\prime}$ such that $\left(i, k^{\prime}\right) \in a$ or $k$ such that $\left(k, j^{\prime}\right) \in a$.

Let $\Omega\left(N, N^{\prime}, v\right)$ be the set of eligible assignments. To find the optimal assignment we need to find $a^{*}$ such that

$$
a^{*} \in \arg \max _{a \in \Omega\left(N, N^{\prime}, v\right)} \sum_{\left(i, j^{\prime}\right) \in a} v_{i j^{\prime}} .
$$

The optimal assignment $a^{*}$ might not be unique and is obtained through the Hungarian algorithm (Kuhn (1955), Munkres (1957)). Without loss of generality, we assume that one of the optimal assignments is to assign $i \in N$ to $i^{\prime} \in N^{\prime}$, for all $i \in N$. We call $\mathcal{V}$ the set of all such matrices. We exploit this feature, and for $i \in N$, it should be understood that $i^{\prime}$ is his assigned match.

An interesting subset of value matrices are those with a dominant diagonal (Solymosi and Raghavan (2001)): $v_{i i^{\prime}} \geq v_{j i^{\prime}}, v_{i j^{\prime}}$ for all $j \in N$ and $j^{\prime} \in N^{\prime}$. Let $\overline{\mathcal{V}}$ be the set of all such matrices.

\subsection{The associated cooperative game and its core}

For every coalition $R \subseteq N, S^{\prime} \subseteq N^{\prime}$, define $v^{R, S^{\prime}}$ as the restriction of $v$ to $R \cup S^{\prime}$. Define $a_{R, S^{\prime}}^{*}$ as (one of) the optimal assignment for the problem $A^{R, S^{\prime}}=\left(R, S^{\prime}, v^{R, S^{\prime}}\right)$. Let $V\left(R \cup S^{\prime}, v\right)=\sum_{\left(i, j^{\prime}\right) \in a_{R, S^{\prime}}^{*}} v_{i j^{\prime}}$ be the maximum value being created by coalition $R \cup S^{\prime}$.

We wish to share that value among the agents. An allocation $y \in \mathbb{R}_{+}^{N \cup N^{\prime}}$ is such that $\sum_{i \in N \cup N^{\prime}} y_{i}=$ $V\left(N \cup N^{\prime}, v\right)$.

The core of problem $A=\left(N, N^{\prime}, v\right)$, labelled as $\operatorname{Core}(A)$, is the set of allocations $y \in \mathbb{R}_{+}^{N \cup N^{\prime}}$ such that

$$
\sum_{i \in R \cup S^{\prime}} y_{i} \geq V\left(R \cup S^{\prime}, v\right) \text { for all } R \subseteq N, S^{\prime} \subseteq N^{\prime}
$$

It is known since Shapley and Shubik (1971) that if $i$ is matched with $i^{\prime}$, then an allocation $y$ that is in the core is such that $y_{i}+y_{i^{\prime}}=v_{i i^{\prime}}$, the value they generate when matched to each other. 
Let $y_{i}^{\max }(v)$ and $y_{i}^{\min }(v)$ be, respectively, the largest and smallest core allocations for agent $i$. From Demange (1982) and Leonard (1983), we know that

$$
\begin{aligned}
& y_{i}^{\max }(v)=V\left(N \cup N^{\prime}, v\right)-V\left(N \backslash i \cup N^{\prime}, v\right) \text { for all } i \in N \\
& y_{i^{\prime}}^{\max }(v)=V\left(N \cup N^{\prime}, v\right)-V\left(N \cup N^{\prime} \backslash i^{\prime}, v\right) \text { for all } i^{\prime} \in N^{\prime} .
\end{aligned}
$$

Combining with $y_{i}+y_{i^{\prime}}=v_{i i^{\prime}}$, we obtain

$$
\begin{aligned}
& y_{i}^{\min }(v)=v_{i i^{\prime}}-V\left(N \cup N^{\prime}, v\right)+V\left(N \cup N^{\prime} \backslash i^{\prime}, v\right) \text { for all } i \in N \\
& y_{i^{\prime}}^{\min }(v)=v_{i i^{\prime}}-V\left(N \cup N^{\prime}, v\right)+V\left(N \backslash i \cup N^{\prime}, v\right) \text { for all } i^{\prime} \in N^{\prime} .
\end{aligned}
$$

We define the Core Selection property as follows:

Core Selection: For all problems $A, y(A) \in \operatorname{Core}(A)$.

\subsection{Solutions}

A solution (or sharing method) $y(A)$ assigns an allocation to every problem $A$. Again, when there is no risk of confusion, we write $y(v)$ instead of $y$.

We introduce the allocations suggested by Demange (1982) and Leonard (1983). Let $y^{N}$ be defined as follows: $y_{i}^{N}(v)=y_{i}^{\max }(v)$ for all $i \in N$ and $y_{i^{\prime}}^{N}(v)=y_{i^{\prime}}^{\min }(v)$ for all $i^{\prime} \in N^{\prime}$. Let $y^{N^{\prime}}(v)$ be defined as follows: $y_{i^{\prime}}^{N^{\prime}}(v)=y_{i^{\prime}}^{\max }(v)$ for all $i^{\prime} \in N^{\prime}$ and $y_{i}^{N^{\prime}}(v)=y_{i}^{\min }(v)$ for all $i \in N$. Following Thompson (1981), we define the Fair Division solution as the average of the allocations most favorable to agents on each side of the market. Formally, let $y^{F D}(v) \equiv \frac{y^{N}(v)+y^{N^{\prime}}(v)}{2}=\frac{y^{\max }(v)+y^{\min }(v)}{2}$. The solution is called the $\tau$-value in Nunez and Rafels (2002).

\section{The assignment problem as a bankruptcy problem}

\subsection{Bankruptcy problems}

Bankruptcy problems are well studied, and consist in one of the purest examples of sharing problems, being appropriate to share an estate among siblings or the cost of a public project among a group that can jointly afford it. Formally, let $M$ be a set of agents. Let $c \in \mathbb{R}^{M}$ be the vector of claims and $E \in \mathbb{R}_{+}$be the endowment, with $E \leq \sum_{i \in M} c_{i}$. A bankruptcy problem is $B=(M, c, E)$. An allocation $\gamma \in \mathbb{R}_{+}^{M}$ is such that $\sum_{i \in M} \gamma_{i}=E$. A sharing method for bankruptcy problems, $\gamma(B)$ assigns an allocation to every bankruptcy problem $B$.

\subsection{Assignment problems as a series of 2-player bankruptcy problems}

Consider an assignment problem $A=\left(N, N^{\prime}, v\right)$, with $v \in \mathcal{V}$. As previously discussed, Core Selection imposes the constraint that $y_{i}+y_{i^{\prime}}=v_{i i^{\prime}}$ for all $i \in N$. We can thus view an assignment problem as a series of bankruptcy problems between pairs of agents $i$ and $i^{\prime}$. As for claims, the obvious candidate is to use the maximal core allocations, as, if we use Core Selection as a focal property, this is the maximum an agent can aspire to. Formally, each assignment problem $A$ can be transformed into a series of bankruptcy problems $B_{i i^{\prime}}^{A}$, one for each pair of agents $\left(i, i^{\prime}\right)$ and such that $B_{i i^{\prime}}^{A}=$ $\left(\left\{i, i^{\prime}\right\},\left\{y_{i}^{\max }(v), y_{i^{\prime}}^{\max }(v)\right\}, v_{i i^{\prime}}\right)$.

\subsection{Solutions for two-player bankruptcy problems}

Note that the set of bankruptcy problems that we obtain from assignment problems is a strict subset of bankruptcy problems: not only are we limited to two-player problems, but we also have, by definition, that $c_{i} \leq E$ for all $i \in M$. Let $\mathcal{B}$ be the set of all such bankruptcy problems. Among the large sets of 
solutions proposed for the assignment problem, we focus on the two-player solutions and adapt them to our set $\mathcal{B}$.

The paper will mostly focus on the Concede-and-Divide solution (Aumann and Maschler (1985)) which consists in first assigning to each agent the difference between the endowment and the claim of the other player, before dividing the remainder equally.

For all $B \in \mathcal{B}, \gamma_{i}^{C D}(B)=E-c_{i^{\prime}}+\frac{c_{i}+c_{i^{\prime}}-E}{2}=\frac{c_{i}-c_{i^{\prime}}+E}{2}$ and similarly $\gamma_{i^{\prime}}^{C D}(B)=\frac{c_{i^{\prime}}-c_{i}+E}{2}$.

Over the set of two-player bankruptcy problems, the Concede-and-Divide solution coincide with many well-studied solutions, notably the Talmud rule (Aumann and Maschler (1985)) and the Random Arrival rule (O’Neill (1982)).

Another popular solution is the Constrained Equal Losses rule, that tries to equalize the loss (claim minus allocation) of each agent. For general two-player problems it is often impossible to equalize the losses, we then prioritize the agent with the larger claim, to bring his loss as close as possible to the loss of the smaller claimant. With our constraint that $c_{i} \leq E$ for all $i \in M$, this is never a problem and we can always achieve equal losses. We thus have that

$$
\begin{aligned}
c_{i}-\gamma_{i}^{C E L} & =c_{i^{\prime}}-\gamma_{i^{\prime}}^{C E L} \\
& =c_{i^{\prime}}-E+\gamma_{i}^{C E L}
\end{aligned}
$$

which simplifies to $\gamma_{i}^{C E L}=\frac{c_{i}-c_{i^{\prime}}+E}{2}=\gamma_{i}^{C D}$. Thus, within $\mathcal{B}$, the Concede-and-Divide and (Constrained) Equal Losses solutions are one and the same.

We complete this section by describing two other popular solutions for bankruptcy problems that do not coincide with Concede-and-Divide within $\mathcal{B}$.

The Constrained Equal Awards rule is such that we want to have allocations as equal as possible, under the constraint that no agent receives more than her claim. Thus, $\gamma_{i}^{C E A}=\min \left\{c_{i}, \lambda\right\}$, with $\lambda$ chosen so that $\gamma_{i}^{C E A}+\gamma_{i^{\prime}}^{C E A}=E$.

The proportional rule divides the endowment in proportion to claims: $\gamma_{i}^{P}=\frac{c_{i}}{c_{i}+c_{i^{\prime}}} E$.

\subsection{Fair Division is Concede-and-Divide}

We conclude this section by showing that the Fair-Division solution for assignment problems is equivalent to the Concede-and-Divide solution for the corresponding bankruptcy problems.

Theorem 1 For all assignment problem $A=\left(N, N^{\prime}, v\right), y_{i}^{F D}(A)=\gamma_{i}^{C D}\left(B_{i i^{\prime}}^{A}\right)$ for all $i \in N$ and $y_{i^{\prime}}^{F D}(A)=\gamma_{i^{\prime}}^{C D}\left(B_{i i^{\prime}}^{A}\right)$ for all $i^{\prime} \in N^{\prime}$.

Proof. Let $A=\left(N, N^{\prime}, v\right)$ and $i \in N$. We have that

$$
\begin{aligned}
\gamma_{i}^{C D}\left(B_{i i^{\prime}}^{A}\right) & =\frac{c_{i}-c_{i^{\prime}}+E}{2} \\
& =\frac{y_{i}^{\max }(v)-y_{i^{\prime}}^{\max }(v)+v_{i i^{\prime}}}{2} \\
& =\frac{y_{i}^{\max }(v)+y_{i}^{\min }(v)}{2} \\
& =\frac{y_{i}^{N}(A)+y_{i}^{N^{\prime}}(A)}{2} \\
& =y_{i}^{F D}(A) .
\end{aligned}
$$

We similarly obtain that $y_{i^{\prime}}^{F D}(A)=\gamma_{i^{\prime}}^{C D}\left(B_{i i^{\prime}}^{A}\right)$ for all $i^{\prime} \in N^{\prime}$. 


\section{The minimal core allocations for assignment problems}

Given the importance in our analysis of the minimal core allocations for the assignment problem, we explore how to obtain these values. We propose a new algorithm to obtain them. Our algorithm has the advantage that it only requires to solve for the optimal assignment of the grand coalition $N \cup N^{\prime}$. By opposition, the previously known method, defined in Section 2, requires to solve for the optimal assignments of coalitions $N \backslash i \cup N^{\prime}$ for all $i \in N$ and $N \cup N^{\prime} \backslash i^{\prime}$ for all $i^{\prime} \in N^{\prime}$.

It is obtained by defining a variant of the Hungarian Algorithm. Furthermore, we show that if we remove these minimal core allocations from the value matrix, the resulting matrix has a dominant diagonal and all agents have a minimal core allocations of zero in this reduced problem.

We need the following definitions: Let $D_{i}(v)=\arg \max _{j^{\prime} \in N^{\prime}} v_{i j^{\prime}}$ for all $i \in N$. In words, $D_{i}(v)$ is the set of agents that create the largest value with $i$. Notice that by definition if $i^{\prime} \in D_{i}(v)$ for all $i \in N$ and $i \in D_{i^{\prime}}(v)$ for all $i^{\prime} \in N^{\prime}$, then $v \in \overline{\mathcal{V}}$.

The algorithm proceeds as follows: our first objective is to make every diagonal element the maximal element on its row. If it's not true, there is an agent $j^{\prime} \in N^{\prime}$ such that $v_{i j^{\prime}}-v_{i i^{\prime}}>0$. We reduce all values created by $j^{\prime}$ by $v_{i j^{\prime}}-v_{i i^{\prime}}$ and repeat the process until that first objective is attained. We then proceed to the second objective, which consists in making every diagonal element the maximal element in its column. When these two objectives are attained ${ }^{1}$, we have a matrix with a dominant diagonal. In addition, the extracted values are exactly the minimal core allocations.

Algorithm 1 Start with an assignment problem $A=\left(N, N^{\prime}, v\right)$.

Step 1: If $i^{\prime} \in D_{i}(v)$ for all $i \in N$, let $e^{1}=0^{N \cup N^{\prime}}, v^{2}=v$ and $K=1$ and proceed to step $K+1$. Otherwise, let $e_{i^{\prime}}^{1}=\max _{j \in N} v_{j i^{\prime}}-v_{j j^{\prime}}$ for all $i^{\prime} \in N^{\prime}, e_{i}^{1}=0$ for all $i \in N$, $v^{2}$ be such that $v_{i j^{\prime}}^{2}=\max \left\{0, v_{i j^{\prime}}-e_{j^{\prime}}^{1}\right\}$ for all $i \in N$ and $j^{\prime} \in N^{\prime}$; and proceed to step 2.

Step $k$, for $1<k \leq K:$ If $i^{\prime} \in D_{i}\left(v^{k}\right)$ for all $i \in N$, let $e^{k}=0^{N \cup N^{\prime}}, v^{k+1}=v^{k}$ and $K=k$ and proceed to step $K+1$. Otherwise, let $e_{i^{\prime}}^{k}=\max _{j \in N} v_{j i^{\prime}}^{k}-v_{j j^{\prime}}^{k}$ for all $i^{\prime} \in N^{\prime}, e_{i}^{1}=0$ for all $i \in N$, $v^{k+1}$ be such that $v_{i j^{\prime}}^{k+1}=\max \left\{0, v_{i j^{\prime}}^{k}-e_{j^{\prime}}^{k}\right\}$ for all $i \in N$ and $j^{\prime} \in N^{\prime}$; and proceed to step $k+1$.

Step $K+1:$ If $i \in D_{i^{\prime}}\left(v^{K+1}\right)$ for all $i^{\prime} \in N^{\prime}$, let $e^{K+1}=0^{N \cup N^{\prime}}, v^{*}=v^{K+1}$ and $K^{\prime}=1$ and terminate. Otherwise, let $e_{i}^{K+1}=\max _{j^{\prime} \in N^{\prime}} v_{i j^{\prime}}^{K+1}-v_{j j^{\prime}}^{K+1}$ for all $i \in N, e_{i^{\prime}}^{1}=0$ for all $i^{\prime} \in N^{\prime}, v^{K+2}$ be such that $v_{i j^{\prime}}^{K+2}=\max \left\{0, v_{i j^{\prime}}^{K+1}-e_{i}^{K+1}\right\}$ for all $i \in N$ and $j^{\prime} \in N^{\prime}$; and proceed to step $K+2$.

Step $K+k^{\prime}$, for $1<k^{\prime} \leq K^{\prime}:$ If $i \in D_{i^{\prime}}\left(v^{K+k^{\prime}}\right)$ for all $i^{\prime} \in N^{\prime}$, let $e^{K+k^{\prime}}=0^{N \cup N^{\prime}}, \bar{v}=v^{K+k^{\prime}}$ and $K^{\prime}=k^{\prime}$ and terminate. Otherwise, let $e_{i}^{K+k^{\prime}}=\max _{j^{\prime} \in N^{\prime}} v_{i j^{\prime}}^{K+k^{\prime}}-v_{j j^{\prime}}^{K+k^{\prime}}$ for all $i \in N, e_{i^{\prime}}^{1}=0$ for all $i^{\prime} \in N^{\prime}, v^{K+k^{\prime}+1}$ be such that $v_{i j^{\prime}}^{K+k^{\prime}+1}=\max \left\{0, v_{i j^{\prime}}^{K+k^{\prime}}-e_{i}^{K+k^{\prime}}\right\}$ for all $i \in N$ and $j^{\prime} \in N^{\prime}$; and proceed to step $K+k^{\prime}+1$.

See Demange (1982) for a similar algorithm. Both $K$ and $K^{\prime}$ are finite numbers and thus the algorithm stops in a finite number of steps $K+K^{\prime}$ and produces the vector $e(v)=\sum_{k=1}^{K+K^{\prime}} e^{k}$ and the matrix $\bar{v} \in \overline{\mathcal{V}}$.

Example 1 Let $N=\{1,2,3,4\}$ and $N^{\prime}=\left\{1^{\prime}, 2^{\prime}, 3^{\prime}, 4^{\prime}\right\}$ and $v$ be as follows:

\begin{tabular}{c|cccc}
$v_{i j^{\prime}}$ & 1 & $2^{\prime}$ & 3 & $4^{\prime}$ \\
\hline 1 & 7 & 6 & 1 & 7 \\
2 & 2 & 3 & 5 & 1 \\
3 & 0 & 2 & 4 & 0 \\
4 & 6 & 2 & 7 & 7
\end{tabular}

\footnotetext{
${ }^{1}$ The two objectives are independent. We can thus change their order. It is also possible to combine some steps in
} the following algorithm. For simplicity and transparency, we have chosen to keep the two objectives separate. 
We can easily see that the optimal assignment is on the diagonal. However, the matrix does not have a dominant diagonal. We have that $V\left(N \cup N^{\prime}, v\right)=21$.

We apply the algorithm.

Step 1: We do not have that $i^{\prime} \in D_{i}(v)$ for all $i \in N$. We have $e_{3^{\prime}}^{1}=v_{23^{\prime}}-v_{22^{\prime}}=2$ and $e_{i}^{1}=0$ for other agents. We have $v^{2}$ as follows:

\begin{tabular}{c|cccc}
$v_{i j^{\prime}}^{2}$ & 1 & 2 & 3 & $4^{\prime}$ \\
\hline 1 & 7 & 6 & 0 & 7 \\
2 & 2 & 3 & 3 & 1 \\
3 & 0 & 2 & 2 & 0 \\
4 & 6 & 2 & 5 & 7
\end{tabular}

Step 2: We have that $i^{\prime} \in D_{i}\left(v^{2}\right)$ for all $i \in N$, so $K=2$ and we proceed to step 3, with $v^{3}=v^{2}$.

Step 3: We do not have that $i \in D_{i^{\prime}}\left(v^{3}\right)$ for all $i \in N$. We have $e_{1}^{3}=v_{12^{\prime}}^{3}-v_{22^{\prime}}^{3}=3, e_{4}^{3}=$ $v_{43^{\prime}}^{3}-v_{33^{\prime}}^{3}=3$ and $e_{i}^{3}=0$ for all other agents. We obtain $v^{4}$ :

\begin{tabular}{c|cccc}
$v_{i j^{\prime}}^{4}$ & 1 & 2 & 3 & 4 \\
\hline 1 & 4 & 3 & 0 & 4 \\
2 & 2 & 3 & 3 & 1 \\
3 & 0 & 2 & 2 & 0 \\
4 & 3 & 0 & 2 & 4
\end{tabular}

Step 4: We do not have that $i \in D_{i^{\prime}}\left(v^{4}\right)$ for all $i \in N$. We have $e_{2}^{4}=v_{23^{\prime}}^{4}-v_{33^{\prime}}^{4}=1$ and $e_{i}^{4}=0$ for all other agents. We obtain $v^{5}$ :

\begin{tabular}{c|cccc}
$v_{i j^{\prime}}^{5}$ & 1 & 2 & 3 & 4 \\
\hline 1 & 4 & 3 & 0 & 4 \\
2 & 1 & 2 & 2 & 0 \\
3 & 0 & 2 & 2 & 0 \\
4 & 3 & 0 & 2 & 4
\end{tabular}

Step 5: We do not have that $i \in D_{i^{\prime}}\left(v^{5}\right)$ for all $i \in N$. We have $e_{1}^{5}=v_{12^{\prime}}^{5}-v_{22^{\prime}}^{5}=1$ and $e_{i}^{5}=0$ for all other agents. We obtain $v^{6}$ :

\begin{tabular}{c|cccc}
$v_{i j^{\prime}}^{6}$ & 1 & 2 & 3 & 4 \\
\hline 1 & 3 & 2 & 0 & 3 \\
2 & 1 & 2 & 2 & 0 \\
3 & 0 & 2 & 2 & 0 \\
4 & 3 & 0 & 2 & 4
\end{tabular}

Step 6: Since $i \in D_{i^{\prime}}\left(v^{6}\right)$ for all $i^{\prime} \in N^{\prime}$, we have $K^{\prime}=4, v^{*}=v^{6}$. Thus, $e_{1}=3+0+1=4$, $e_{2}=0+1+0=1, e_{3}=0$ and $e_{4}=3+0+0=3$, as well as $e_{1^{\prime}}=0, e_{2^{\prime}}=0, e_{3^{\prime}}=2$ and $e_{4^{\prime}}=0$. Notice that $\bar{v} \in \overline{\mathcal{V}}$.

It turns out that the vector $e(v)$ is the vector of minimal core allocations. To show this result formally, we need the following lemma, that describes how the core behaves when we subtract from the values a vector that is no larger than the vector of minimal core allocations.

Lemma 1 For all $a \leq y^{\min }(v)$ and $v^{-a}$ such that $v_{i j^{\prime}}^{-a}=\max \left\{0, v_{i j^{\prime}}-a_{i}-a_{j^{\prime}}\right\}$ for all $i \in N, j^{\prime} \in N^{\prime}$, we have that $\operatorname{Core}(v)=\operatorname{Core}\left(v^{-a}\right)+a$ and $y^{\min }(v)=y^{\min }\left(v^{-a}\right)+a$.

Proof. Per Shapley and Shubik (1971), the relevant core constraints are those for pairs of agents. We thus have that $x \in \operatorname{Core}(v)$ if $x_{k}+x_{l^{\prime}} \geq v_{k l^{\prime}}$ for all $k \in N, l^{\prime} \in N^{\prime}$.

Nunez and Rafels (2006) show that if $v_{i j^{\prime}}-a_{i}-a_{j^{\prime}} \geq 0$, we have that $x_{i}+x_{j^{\prime}} \geq v_{i j^{\prime}} \Leftrightarrow x_{i}-a_{i}+$ $x_{j^{\prime}}-a_{j^{\prime}} \geq v_{i j^{\prime}}^{-a}$ We show that it is also true if $v_{i j^{\prime}}-a_{i}-a_{j^{\prime}}<0$.

By definition of $y^{\min }$, if $x \in \operatorname{Core}(v)$, then $x \geq y^{\min }(v)$. We thus have that $x_{i}+x_{j^{\prime}} \geq y_{i}^{\min }(v)+$ $y_{j^{\prime}}^{\min }(v)$ and by assumption $y_{i}^{\min }(v)+y_{j^{\prime}}^{\min }(v) \geq a_{i}+a_{j^{\prime}}>v_{i j^{\prime}}$. We thus also have that $x_{i}-a_{i}+x_{j^{\prime}}-a_{j^{\prime}} \geq$ $0=v_{i j}^{-a}$ and thus $x-a \in \operatorname{Core}\left(v^{-a}\right)$. 
It remains to show that $x-a \in \operatorname{Core}\left(v^{-a}\right) \Rightarrow x \in \operatorname{Core}(v)$. If $x-a \in \operatorname{Core}\left(v^{-a}\right)$, then for all $i \in N$ and $j^{\prime} \in N^{\prime}, x_{i}-a_{i}+x_{j^{\prime}}-a_{j^{\prime}} \geq v_{i j^{\prime}}^{-a}=\max \left\{0, v_{i j^{\prime}}-a_{i}-a_{j^{\prime}}\right\}$. This can be written as

$$
x_{i}+x_{j^{\prime}} \geq \max \left\{a_{i}+a_{j^{\prime}}, v_{i j^{\prime}}\right\} \geq v_{i j^{\prime}}
$$

and thus $x \in \operatorname{Core}(v)$.

Thus, $x \in \operatorname{Core}(v)$ iff $x-a \in \operatorname{Core}\left(v^{-a}\right)$. This implies that $\operatorname{Core}(v)=\operatorname{Core}\left(v^{-a}\right)+a$ and $y^{\min }(v)=y^{\min }\left(v^{-a}\right)+a$.

We are now ready to show that $e(v)$ obtained in our algorithm is equal to $y^{\min }(v)$.

Theorem 2 For any $v \in \mathcal{V}, e(v)=y^{\min }(v)$.

Proof. Suppose that at step $k$ in the above algorithm, we have $e_{i^{\prime}}^{k}=v_{j i^{\prime}}^{k}-v_{j j^{\prime}}^{k}$. It implies that we have $V\left(\left\{j, j^{\prime}\right\}, v^{k}\right)=v_{j j^{\prime}}^{k}$ and $V\left\{\left(j, i^{\prime}, j^{\prime}\right\}, v^{k}\right)=v_{j i^{\prime}}^{k}$. The core constraints imposes $y_{j}+y_{i^{\prime}}+y_{j^{\prime}} \geq v_{j i^{\prime}}^{k}$ and $y_{j}+y_{j^{\prime}}=v_{j j^{\prime}}^{k}$. Combining, we obtain $y_{i^{\prime}} \geq v_{j i^{\prime}}^{k}-v_{j j^{\prime}}^{k}$. The minimal core allocation for agent $i^{\prime}$ is thus at least as large as $v_{j i^{\prime}}^{k}-v_{j j^{\prime}}^{k}$. Since the core is non-empty, we cannot have the minimal core allocation to be smaller than this bound. Thus, at each stage $k, e^{k} \leq y^{\min }\left(v^{k}\right)$.

By Lemma 1, we obtain that at each stage, Core $\left(v^{k}\right)=\operatorname{Core}\left(v^{k+1}\right)+e^{k}$ and $y^{\min }\left(v^{k}\right)=y^{\min }\left(v^{k+1}\right)+$ $e^{k}$. At stage $K+K^{\prime}$, we have, by definition, that $y^{\min }(\bar{v})=0^{N \cup N^{\prime}}$ and thus $y^{\min }\left(v^{K+K^{\prime}-1}\right)=e^{K+K^{\prime}-1}$. We then obtain $y^{\min }\left(v^{K+K^{\prime}-2}\right)=y^{\min }\left(v^{K+K^{\prime}-1}\right)+e^{K+K^{\prime}-2}=e^{K+K^{\prime}-1}+e^{K+K^{\prime}-2}$. In the same manner, for all $k^{\prime}=1, \ldots, K^{\prime}$ we obtain $y^{\min }\left(v^{K+K^{\prime}-k^{\prime}}\right)=\sum_{l^{\prime}=1}^{k^{\prime}} e^{K+K^{\prime}-l^{\prime}}$. Once again by the same procedure, we obtain, for all $k=1, \ldots, K-1$ that

$$
y^{\min }\left(v^{K-k}\right)=\sum_{l=1}^{k} e^{K-k}+\sum_{l^{\prime}=1}^{K^{\prime}} e^{K+K^{\prime}-l^{\prime}} .
$$

In particular, for $k=K-1$, we obtain $y^{\min }(v)=\sum_{l=1}^{K-1} e^{K-k}+\sum_{l^{\prime}=1}^{K^{\prime}} e^{K+K^{\prime}-l^{\prime}}=e(v)$.

\section{Characterizations of the Fair Division Solution}

We now introduce a series of properties that will be used to characterize the Fair Division Solution. We start with a simple symmetry property. Suppose that agents $i \in N$ and $i^{\prime} \in N^{\prime}$ are symmetric in the following sense: the value created by $i$ and agent $k^{\prime}$ is exactly the same as the one obtained by $i^{\prime}$ and $k$, for all $k \in N$. Then, agents should receive the same allocation.

Symmetry: For all $A=\left(N, N^{\prime}, v\right)$, if $v$ is such that $v_{k i^{\prime}}=v_{i k^{\prime}}$ for all $k \in N$, then $y_{i}(v)=y_{i^{\prime}}(v)$.

It is natural to have shares depend on the outside options, which in our case are the matching values with partners other than the assigned partner. In particular, an agent should be assigned a larger share if many agents on the other side want to be matched with him, because he creates high values with many partners. In those cases, the threat that the agent can defect from the optimal assignment is credible. However, in the case in which a pair of matched agents cannot create larger values with other partners, than the threat that he will defect is empty. We argue that when the value matrix changes one possibility is that i) a pair of agents are matched together in both situations and ii) their threats to deviate with other partners are empty in both situations. We argue that when these two conditions are met, for all the pairs of matched agents, the situations are identical and the allocations should not change with the changes in the value matrix.

Independence of Empty Threats. If $v$ and $\tilde{v}$ are such that $v_{i i^{\prime}}=\tilde{v}_{i i^{\prime}} \geq v_{i j^{\prime}}, v_{j i^{\prime}}, \tilde{v}_{i j^{\prime}}, \tilde{v}_{j i^{\prime}}$ for all $i, j \in N$, then $y(v)=y(\tilde{v})$.

In particular, Independence of Empty Threats states that if two matrices with dominant diagonals have identical values on the diagonal, then the shares should be the same, as elements outside the diagonal (the so-called empty threats) are irrelevant. 
Our final two properties take advantage of the link between assignment and bankruptcy problems, as discussed in Section 3. The first is based on the Minimal Rights First property for bankruptcy problems (Curiel, Maschler, Tijs (1987). The Minimal Rights First property considers the case in which everybody but agent $i$ has received their full claims. If the resource has not been exhausted, then, agent $i$ should clearly receive what is left, agent $i$ 's so-called minimal right. The property then says that we should obtain the same shares if we i) apply our solution to the original problem or ii) assign everybody their minimal right, reduce their claims and the endowment accordingly and apply our solution to the new problem. The equivalent concept for assignment problems is for shares to be invariant to our treatment of the minimal core allocations: we obtain the same shares if we i) apply our solution to the original problem or ii) subtract from all values the minimal core allocations (with censorship to zero if needed) and apply our solution to the new assignment problem.

Minimal Core Allocations First: For any $v \in \mathcal{V}, y(v)=y(\bar{v})+y^{\min }(v)$.

Our final property adapts the Reasonable Lower Bound property of Moreno-Ternero and Villar (2004) and Yeh (2008). In the context of the two-player bankruptcy problem, the property says that if an agent claims at most the full endowment, he should receive at least half of its claim. Translated for the assignment problem, we obtain a property always requiring (since claims are never larger than the endowment) that an agent receives at least half of his maximal core allocation.

Reasonable Lower Bound: For any $v \in \mathcal{V}$ we have $y(v) \geq \frac{y^{\max }(v)}{2}$.

We are now ready for our first characterization.

Theorem 3 A solution y satisfies Symmetry, Core Selection, Independence of Empty Threats and Minimal Core Allocations First if and only if $y=y^{F D}$.

Proof. We first show that $y^{F D}$ satisfies the properties.

Symmetry: Suppose that $v$ is such that $v_{k i^{\prime}}=v_{i k^{\prime}}$ for all $k \in N$. Then, $y_{i}^{\min }(v)=y_{i^{\prime}}^{\min }(v)$ and $y_{i}^{\max }(v)=y_{i^{\prime}}^{\max }(v)$, yielding that $y_{i}^{F D}(v)=y_{i^{\prime}}^{F D}(v)$.

Core Selection: By Lemma 1, we only need to show that it is satisfied for $v \in \overline{\mathcal{V}}$. Following Shapley and Shubik (1971), we only need to verify coalitions consisting of one player on each side of the market. Take $i \in N$ and $j^{\prime} \in N^{\prime} . y_{i}^{F D}(v)+y_{j^{\prime}}^{F D}(v)=\frac{v_{i i^{\prime}}}{2}+\frac{v_{j j^{\prime}}}{2} \geq v_{i j^{\prime}}$, since, by definition of $\overline{\mathcal{V}}, v_{i i^{\prime}}, v_{j j^{\prime}} \geq v_{i j^{\prime}}$.

Independence of Empty Threats: Let $v$ and $\tilde{v}$ be such that $v_{i i^{\prime}}=\tilde{v}_{i i^{\prime}} \geq v_{i j^{\prime}}, v_{j i^{\prime}}, \tilde{v}_{i j^{\prime}}, \tilde{v}_{j i^{\prime}}$ for all $i, j \in N$. Then, $y_{i}^{F D}(v)=\frac{v_{i i^{\prime}}}{2}=\frac{\tilde{v}_{i i^{\prime}}}{2}=y_{i}^{F D}(\tilde{v})$ for all $i \in N$. We obtain the same result for $i^{\prime} \in N^{\prime}$.

Minimal Core Allocations First: For all $i \in N$ we have:

$$
\begin{aligned}
y_{i}^{F D}(v) & =\frac{y_{i}^{\max }(v)+y_{i}^{\min }(v)}{2} \\
& =\frac{y_{i}^{\max }(\bar{v})+y_{i}^{\min }(\bar{v})}{2}+y_{i}^{\min }(v) \\
& =y_{i}^{F D}(\bar{v})+y_{i}^{\min }(v),
\end{aligned}
$$

where the second equality comes from Lemma 1 . We obtain the same result for $i^{\prime} \in N^{\prime}$.

We show that a unique solution satisfies the properties. Suppose that $y$ satisfies all properties.

By Minimal Core Allocations First, $y(v)=y(\bar{v})+y^{\min }(v)$. Let $\bar{v}^{0}$ be such that $\bar{v}_{i i^{\prime}}^{0}=\bar{v}_{i i^{\prime}}$ for all $i \in N$ and $\bar{v}_{i j^{\prime}}^{0}=0$ if $i \neq j$. By construction, $\bar{v} \in \overline{\mathcal{V}}$. By Independence of Empty Threats, we have that $y(\bar{v})=y\left(\bar{v}^{0}\right)$. By Core Selection, we have that $y_{i}\left(\bar{v}^{0}\right)+y_{i^{\prime}}\left(\bar{v}^{0}\right)=\bar{v}_{i i^{\prime}}^{0}=\bar{v}_{i i^{\prime}}$ for $i=1, \ldots, n$. By Symmetry, we must have that $y_{i}\left(\bar{v}^{0}\right)=y_{i^{\prime}}\left(\bar{v}^{0}\right)$. Combining these two results, we obtain $y_{i}\left(\bar{v}^{0}\right)=y_{i^{\prime}}\left(\bar{v}^{0}\right)=\frac{\bar{v}_{i i^{\prime}}}{2}$ for $i=1, \ldots, n$. Thus, we have a unique solution defined for all $v \in \mathcal{V}$.

Notice that we barely use Core Selection in the proof. We can replace it by the weaker property of Submarket Efficiency (van den Brink and Pinter (2015)), which says that if we can divide $N \cup N^{\prime}$ in subgroups such that sellers in one subgroup have no gains to match to buyers in other groups, and vice-versa, then the members of each subgroup will split the value they jointly generate. Since we focus on the core in our analysis, we use the stronger Core Selection property.

We can also forego Core Selection, as well as Independence of Empty Threats, if we are ready to strengthen Symmetry. We replace it with Reasonable Lower Bound, which, in opposition to Symmetry, 
applies to all matrices and suggests some form of symmetry. This second characterization closely follows the characterization of the Concede-and-Divide solution for bankruptcy problems obtained by Yeh (2008).

Theorem 4 A solution y satisfies Reasonable Lower Bound and Minimal Core Allocations First if and only if $y=y^{F D}$.

Proof. We have shown in Theorem 3 that $y^{F D}$ satisfies Minimal Core Allocations First. We show that it satisfies Reasonable Lower Bound. We have that $y^{F D}=\frac{y^{\max }(v)+y^{\min }(v)}{2} \geq \frac{y^{\max }(v)}{2}$ since $y^{\min }(v) \geq$ $0^{N \cup N^{\prime}}$.

We show that a unique solution satisfies the properties. Suppose that $y$ satisfies Reasonable Lower Bound and Minimal Core Allocations First. By Minimal Core Allocations First, $y(v)=y(\bar{v})+y^{\min }(v)$. By definition, $y^{\min }(\bar{v})=0^{N \cup N^{\prime}}$ and thus, for all $i \in N, y_{i}^{\max }(\bar{v})=y_{i^{\prime}}^{\max }(\bar{v})=\bar{v}_{i i^{\prime}}$. For all $i \in N$ we have $y_{i}(\bar{v}), y_{i^{\prime}}(\bar{v}) \geq \frac{\bar{v}_{i i^{\prime}}}{2}$, by Reasonable Lower Bound, and $\sum_{i \in N} y_{i}(\bar{v})+\sum_{i^{\prime} \in N^{\prime}} y_{i^{\prime}}(\bar{v})=\sum_{i \in N} \bar{v}_{i i^{\prime}}$, by budget balance. The only way to reconcile these two constraints is to have $y_{i}(\bar{v})=y_{i^{\prime}}(\bar{v})=\frac{\bar{v}_{i i^{\prime}}}{2}$ for all $i \in N$. Thus, we have a unique solution defined for all $v \in \mathcal{V}$.

One could obtain the characterization, with the same proof, using a slightly different property proposed by Moreno-Ternero and Villar (2006): if an agent claims the full endowment, he should receive at least half of the endowment. Translated to our problem, it means that if an agent's maximal core allocation is equal to the full value created with his match, he should receive at least half of that value.

\section{Discussion}

\subsection{Matrices with a doubly-dominant diagonals}

It is worth noting the Fair Division solution has some similarity with the one proposed by Nunez and Rafels (2006). Their idea is to transform a matrix $v$ into one with a doubly-dominant diagonal, a property that implies that $i$ ) $v$ has a dominant diagonal and $i i) v$ is buyer-seller exact. A matrix is buyer-seller exact if raising any entry changes the core. They proceed by first transforming $v$ into $v^{b s e}$, the only buyer-seller exact matrix that generates the same core as $v$. They obtain $v^{b s e}$ as follows. For all $i \in N$ and $j^{\prime} \in N^{\prime}$,

$$
v_{i j^{\prime}}^{b s e}=v_{i i^{\prime}}+v_{j j^{\prime}}+V\left(N \backslash j \cup N^{\prime} \backslash i^{\prime}, v\right)-V\left(N \cup N^{\prime}, v\right) .
$$

They then further transform the matrix into a matrix with a doubly-dominant diagonal, $v^{d d}$, by subtracting the minimal core allocations: For all $i \in N$ and $j^{\prime} \in N^{\prime}, v_{i j^{\prime}}^{d d}=v_{i j^{\prime}}^{b s e}-y_{i}^{\min }(v)-y_{j^{\prime}}^{\min }(v)$. Notice that since in moving from $v$ to $v^{b s e}$ we have increased values, there is no need anymore to censor $v_{i j^{\prime}}^{d d}$ to zero, as $v_{i j^{\prime}}^{b s e} \geq y_{i}^{\min }(v)+y_{j^{\prime}}^{\min }(v)$. The main reason for Nunez and Rafels to transform $v$ into $v^{d d}$ is that the Shapley value of a game generated by a matrix with a doubly-dominant diagonal is always in the core. Nunez and Rafels thus propose the allocation $y^{N R}(v)=y^{\min }(v)+S h\left(v^{d d}\right)$. For our purposes, since we do not use the Shapley value, the first part of the operation, in which we transform a matrix into a buyer-seller matrix, is unnecessary. We contrast our method with the translated Shapley value of Nunez and Rafels (2006) in the following example.

Example 2 We consider a simpler version of the above example, in which we remove agents 4 and $4^{\prime}$. We have

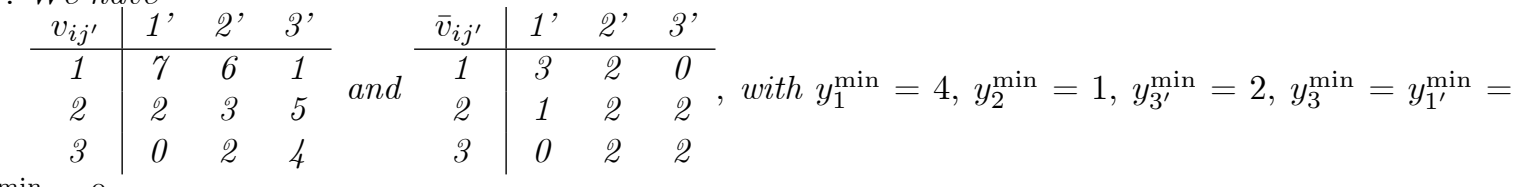

$y_{2^{\prime}}^{\min }=0$. 


We obtain $\bar{v}^{d d}$ as follows:
\begin{tabular}{c|ccccc}
$\bar{v}_{i j^{\prime}}^{d d}$ & 1 & 2 & 3 & \\
\hline 1 & 3 & 2 & 2 \\
2 & 1 & 2 & 2 & \\
2 & We thus obtain the following shares: \\
3 & 1 & 2 & 2 & & \\
& $y^{F D}(\bar{v})$ & $y^{N R}(\bar{v})$ & $y^{F D}(v)$ & $y^{N R}(v)$ \\
1 & 1.5 & $1.61 \overline{6}$ & 5.5 & $5.61 \overline{6}$ \\
2 & 1 & $0.9 \overline{3}$ & 2 & $1.9 \overline{3}$ \\
3 & 1 & $0.9 \overline{3}$ & 1 & $0.9 \overline{3}$ \\
1 & 1.5 & $1.38 \overline{3}$ & 1.5 & $1.38 \overline{3}$ \\
2 & 1 & $1.0 \overline{6}$ & 1 & $1.0 \overline{6}$ \\
3 & 1 & $1.0 \overline{6}$ & 3 & $3.0 \overline{6}$
\end{tabular}

Consider the following interpretation of the methods, that shows how close the two methods are. It turns out that to obtain the matrix with a doubly-dominant diagonal needed for $y^{N R}$, we can also reverse the order of the operations, first removing the minimal core allocations before making the matrix buyer-seller exact. Therefore, we can view both methods as first transforming the matrix into one with a dominant diagonal by extracting the minimal core allocations. To obtain $y^{N R}$, we then transform the matrix into one with a doubly-dominant diagonal, by making changes to off-diagonal elements to obtain buyer-seller exactness, before taking the Shapley value of the resulting game. To obtain the Fair Division solution we transform the off-diagonal elements by making them all equal to zero. Computing the Shapley value of the corresponding cooperative game is equivalent to sharing $v_{i i^{\prime}}$ equally among the two partners. The difference between the two methods can thus be seen as the transformation made to the matrix with a dominant diagonal before applying the Shapley value.

Independence of Empty Threat, used to characterize the Fair Division solution, says that when agents have nothing to gain from deviations, these threats can be seen as empty. It is thus natural to consider the values outside of the optimal assignment as irrelevant for the shares of the agents. Notice that this is in direct opposition with the usual interpretation of the Shapley value, for which all threats are considered as non-empty and relevant, even if they generate much less value. But the core of the assignment problem has two distinctive properties: a pair of matched agents share among themselves the value they generate, and as long as all shares are non-negative, we only need to worry about deviations from pairs. It is because of these characteristics that we can pinpoint much more precisely the threats of deviations than in general cooperative games. This is illustrated clearly by the link with the 2-player bankruptcy problem, in which, by definition, there is nobody to deviate with. Therefore, we believe that our approach is a relevant alternative to allocations based on the Shapley value, such as the method proposed by Nunez and Rafels (2006).

\subsection{Removing Symmetry and extreme points of the core}

If we remove Symmetry from Theorem 3, we obtain many other allocations, including the extreme points of the core of Demange (1982) and Leonard (1983), which jointly maximize the welfare of one side of the market. Symmetry implies that both sides of the market are treated in the same manner, which might not always be true. Note however that if we amend the property to recognize that one side of the market has a systematic advantage, it still would not be enough to characterize the full core, as it also includes allocations in which one side of the market is favored in one pair, but disfavored in another.

\subsection{Other potential links with the bankruptcy problem}

While the Concede-and-Divide solution has a nice interpretation as a solution to the assignment problems, what about the other popular solutions for two-player bankruptcy problems? It turns out to be very difficult to satisfy Core Selection, as seen in the following example. 
Example 3 We consider again the problem of Example 2. We find the following shares for the Constrained Equal Awards and Proportional solutions:

$\begin{array}{ccc} & \gamma^{C E A} & \gamma^{P} \\ 1 & 4 & 4.9 \\ 2 & 1.5 & 1.8 \\ 3 & 2 & 1 . \overline{3} \\ 1^{\prime} & 3 & 2.1 \\ 2^{\prime} & 1.5 & 1.2 \\ 3^{\prime} & 2 & 2 . \overline{6}\end{array}$

See that $v_{23^{\prime}}=5$, but that $\gamma_{2}^{C E A}+\gamma_{3^{\prime}}^{C E A}=3.5$ and $\gamma_{2}^{P}+\gamma_{3^{\prime}}^{P}=4.4 \overline{6}$. Thus, they do not satisfy Core Selection.

This is particularly problematic, as the link between assignment and bankruptcy problems was obtained with Core Selection at the heart of the argument. This is also not quite surprising, as when we move from a $N \times N^{\prime}$ assignment problem to a 2-player bankruptcy problem, we ignore most of the information of the original problem. That the Concede-and-Divide solution is able to generate an allocation in the core regardless of that is noteworthy. There does not seem to be any other solution for bankruptcy problems that can do the same.

There are many other axiomatizations of the Concede-and-Divide solution for 2-player bankruptcy problems, notably offered by Moreno-Ternero and Villar (2004, 2006). Most of the other properties used are difficult to adapt to our context. Many are consistency properties that apply when the endowment changes, all else equal, or when a claim changes, all else equal. This is difficult to use in our context since claims and endowment usually move together. Another difficulty is that many characterizations use self-duality, a property that makes it such that it does not matter if we allocate the endowment or the losses with respect to the claims. In our case, we lose the property that claims are no larger than the (adjusted) endowment in the dual problem.

\subsection{Independence of the properties}

Lemma 2 The properties of Theorem 3 are independent.

Proof. Let $y^{1}$ be defined as follows. If $v \in \overline{\mathcal{V}}$ or if $v_{k i^{\prime}}=v_{i k^{\prime}}$ for $k=1, . ., n$, then $y_{i}^{1}(v)=y_{i^{\prime}}^{1}(v)=\frac{v_{i i^{\prime}}}{2}$ for $i=1, \ldots, n$. Otherwise, $y^{1}(v)=y^{N R}(v)$.

We can verify that $y^{1}$ satisfies Symmetry, Core Selection and Independence of Empty Threats but fails Minimal Core Allocations First.

We can verify that $y^{N R}$ satisfies Symmetry, Core Selection and Minimal Core Allocations First but fails Independence of Empty Threats.

Let $y^{2}$ be defined as follows. If $v \in \overline{\mathcal{V}}$, then $y_{i}^{2}(v)=y_{i^{\prime}}^{2}(v)=\frac{V\left(N \cup N^{\prime}, v\right)}{2 n}$ for $i=1, \ldots, n$. If $v \in \mathcal{V} \backslash \overline{\mathcal{V}}$, let $y^{2}(v)=y^{2}(\bar{v})+y^{\min }(v)$.

We can verify that $y^{2}$ satisfies Symmetry, Independence of Empty Threats and Minimal Core Allocations First but fails Core Selection.

We can verify that $y^{N}$ (and $y^{N^{\prime}}$ ) satisfies Core Selection, Independence of Empty Threats and Minimal Core Allocations First but fails Symmetry.

The following table summarizes the lemma: ("+" indicates that the property is satisfied and "-" that it is not)

$\begin{array}{lllll} & \text { SYM } & \text { CS } & \text { IET } & \text { MCAF } \\ y^{F D} & + & + & + & + \\ y^{1} & + & + & + & - \\ y^{N R} & + & + & - & + \\ y^{2} & + & - & + & + \\ y^{N} & - & + & + & + \\ y^{N^{\prime}} & - & + & + & +\end{array}$


Lemma 3 The properties of Theorem 4 are independent.

Proof. We can verify that $y^{N R}$ satisfies Minimal Core Allocations First but fails Reasonable Lower Bound.

Let $y^{3}$ be defined as follows: $y_{i}^{3}(v)=y_{i^{\prime}}^{3}=\frac{v_{i i^{\prime}}}{2}$ for all $v \in \mathcal{V}$ and all $i \in N$. We can verify that $y^{3}$ satisfies Reasonable Lower Bound but fails Minimal Core Allocations First.

\section{References}

R. Aumann and M. Maschler (1985), Game Theoretic Analysis of a Bankruptcy Problem from the Talmud. Journal of Economic Theory 36, pp. 195-213.

M.L. Balinski and D. Gale (1987), "On the Core of the Assignment Game", in Functional Analysis, Optimization and Mathematical Economics. Oxford University Press, New York, pp. 274-289.

I.J. Curiel, M. Maschler and S.H. Tijs (1987), Bankruptcy Games, Zeitschrift far Operations Research 31, pp. 143-159.

G. Demange (1982), "Strategyproofness in the assignment market game", Laboratoire d'Économie de l'École Polytechnique, Paris, mimeo.

H. Hamers, F. Klijn, T. Solymosi, S. Tijs, J.P. Villar (2002), Assignment games satisfy the CoMaproperty, Games and Economic Behavior 38, pp. 231-239.

H. Kuhn (1955), The Hungarian Method for the Assignment Problem, Naval Research Logistics Quarterly 2, pp. 83-97.

H.B. Leonard (1983), Elicitation of Honest Preferences for the Assignment of Individuals to Positions, Journal of Political Economy 91, pp. 461-479.

J. Moreno-Ternero and A. Villar (2004), The Talmud Rule and the Securement of Agents, Mathematical Social Sciences 47, pp. 245-257.

J. Moreno-Ternero and A. Villar (2006), New Characterizations for a Classical Bankruptcy Rule, Review of Economic Design 10, pp. 73-84.

J. Munkres (1957), Algorithms for the Assignment and Transportation Problems, Journal of the Society for Industrial and Applied Mathematics 5, pp. 32-38.

M. Nunez and C. Rafels (2002), The Assignment Game: the $\tau$-value. International Journal of Game Theory 31, pp. 411-422.

M. Nunez and C. Rafels (2003), Characterization of the Extreme Core Allocations of the Assignment Game, Games and Economic Behavior 44, pp. 311-331.

M. Nunez and C. Rafels (2006), A Canonical Representation for the Assignment Game: the Kernel and the Nucleolus, Barcelona Economics Working Paper 279, 23p.

B. O'Neill (1982), A Problem of Rights Arbitration from the Talmud. Mathematical Social Sciences 2, pp. 345-371.

A.E. Roth and M.A.O. Sotomayor (1992), "Two-Sided Matching: A Study in Game-Theoretic Modeling and Analysis", Econometric Society Monographs, Cambridge University Press, 280p.

L.S. Shapley and M. Shubik (1971), The Assignment Game I: The Core, International Journal of Game Theory 1, pp. 9-25. 
T. Solymosi and T.E.S. Raghavan (2001), Assignment Games with Stable Cores, International Journal of Game Theory 30, pp. 177-185.

G.L. Thompson (1981), "Auctions and Market Games", in Essays in Game Theory and Mathematical Economics in Honor of Oskar Morgenstern, ed. by R. Aumann.

W. Thomson (2003), Axiomatic and game-theoretic analysis of bankruptcy and taxation problems: a survey. Mathematical Social Sciences 45, pp. 249-297.

W. Thomson (2015), Axiomatic and game-theoretic analysis of bankruptcy and taxation problems: an update. Mathematical Social Sciences 74, pp. 41-59.

S.H. Tijs (1981), "Bounds for the core and the $\tau$-value", in Game Theory and Mathematical Economics, ed. by O. Moeschlin and D. Pallaschke, North Holland Publishing Company.

R. van den Brink and M. Pinter (2015), On Axiomatizations of the Shapley Value for Assignment Games, Journal of Mathematical Economics 60, pp. 110-114..

C.-H. Yeh (2008), Secured Lower Bound, Composition Up and Minimal Rights First for Bankruptcy Problems, Journal of Mathematical Economics 44, pp. 925-932. 